$1-1-2021$

\title{
Overview: Literature review of linkage, adherence, and retention interventions for seropositive children and young people in Africa
}

Project SOAR

Follow this and additional works at: https://knowledgecommons.popcouncil.org/departments_sbsr-hiv

Part of the Public Health Commons

How does access to this work benefit you? Let us know!

\section{Recommended Citation}

Project SOAR. 2021. "Overview: Literature review of linkage, adherence, and retention interventions for seropositive children and young people in Africa," Project SOAR Results Brief. Washington, DC: Population Council. 
Overview: Literature review of linkage, adherence, and retention interventions for seropositive children and young people in Africa

In the era of the UNAIDS 95-95-95 goals, HIV testing, engagement in HIV care, and viral suppression, achieved through proper adherence to antiretroviral treatment (ART), are crucial steps toward controlling the HIV epidemic. Considerable work has already been conducted on HIV testing and related interventions to identify HIVpositive infants ( $<1$ year); children (1-9 years); adolescents (10-19 years); and youth (20-24 years). However, these children and young people living with HIV are less likely to be linked to care and adherent to ART, compared to adults. ${ }^{1}$ Furthermore, there has been less focus, to date, on adolescents in the development, implementation, and evaluation of interventions to facilitate ART delivery, retention in care, and adherence to treatment.

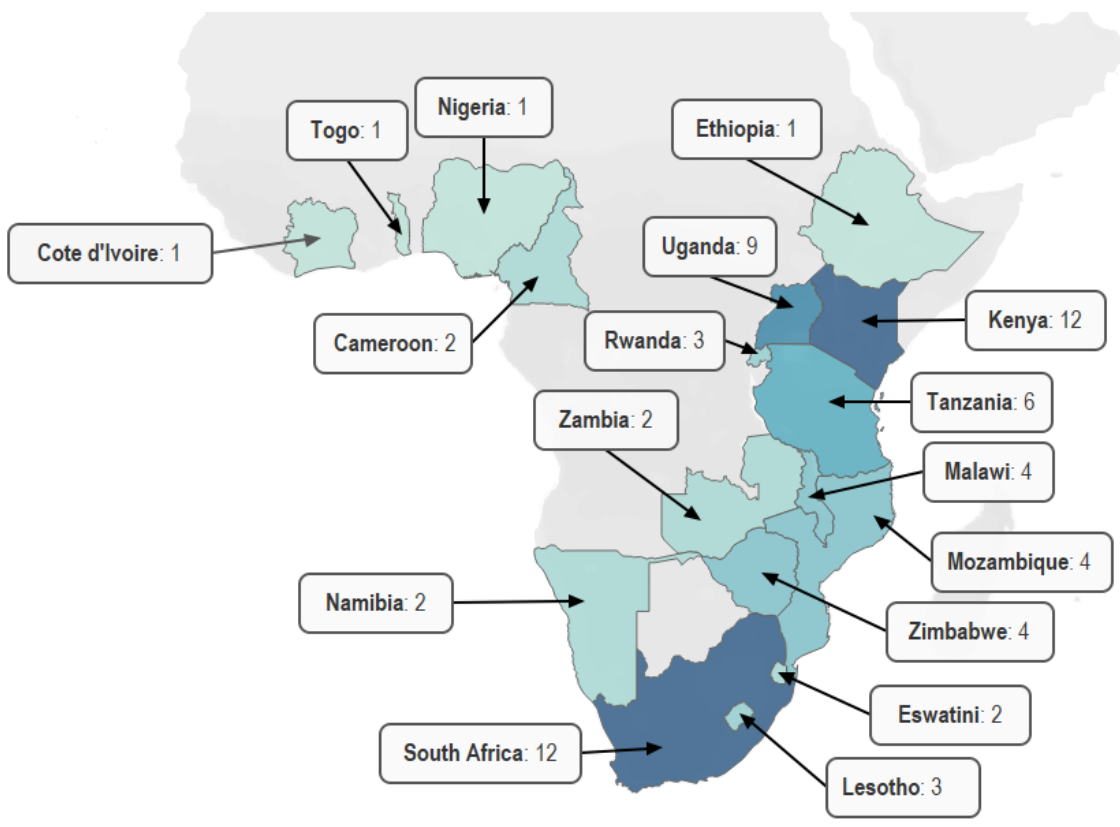

Nevertheless, various strategies have been implemented in countries around the world to reduce barriers to engagement in care and adherence to ART among these vulnerable communities, and to support young people living with HIV to achieve viral suppression. Strategies to engage people living with HIV in care and to improve ART adherence can be categorized as: 1) individual-level interventions, such as information and communication technology-enhanced solutions or education and counselling; 2) group-level interventions, such as community support groups; and 3) policy or systems interventions, such as changes to healthcare delivery models.

Despite these diverse programmatic strategies, a comprehensive understanding of what works best for children and adolescents in sub-Saharan Africa is lacking. To address this gap, Project SOAR conducted a systematic review of the peer-reviewed literature to: 1) document interventions that support engagement in care and ART adherence for infants, children, adolescents, and youth living with HIV in sub-Saharan Africa (SSA), including orphans and other vulnerable children; and 2) inform future policy decisions.

Number of interventions by country, sub-Saharan Africa

\section{METHODS}

We conducted a comprehensive literature search of online databases to identify relevant articles published from 2010 through 2018. The search included keywords focusing on terms used to describe orphaned and vulnerable populations living with HIV in subSaharan African nations.

We developed eligibility criteria with respect to the population, outcomes of interest, and publication type. Studies were eligible for inclusion if they presented an intervention to improve linkage to care, retention, adherence to ART or viral suppression among individuals who were under the age of 18 and living with HIV in sub-Saharan Africa. Using these criteria, the research team independently screened titles and abstracts for inclusion.

\section{RESULTS}

We identified a total of 5,012 unique articles through database searching. Of those, we excluded 4,943 articles during the screening phases, leaving 69 
articles that met all eligibility criteria for inclusion. Of those, 25 studies reported ART initiation as an outcome, 36 reported on retention in HIV care and/ or ART adherence as an outcome, and 12 reported on viral suppression as an outcome. In cases where study populations included those under as well as over age 18 , we included findings pertaining to these older participants (i.e., youth up to age 24).

Table 1 (on page 4) provides a summary of experimental studies included in the systematic review. An Excel spreadsheet summarizing all studies included in the systematic review can be found on the Project SOAR website at: https://projsoar.org/wp-content/ uploads/2021/01/ChldrnYngPpl_LinkageRetention_ LitReviewMatrix.xlsx

\section{ART initiation as an outcome}

Total number of included studies: 25

Populations targeted: infants (12); children (15); adolescents (14); and youth (6)

Study designs: non-experimental and no comparison group (20); non-experimental with a comparison group (3); and experimental (2)

\section{Effectiveness:}

- Diagnostic testing interventions for infants, such as point-of-care PCR testing and electronic patient monitoring systems, were effective at shortening the time until diagnosis, and increasing the number of patients who both received results and, when appropriate, initiated ART. ${ }^{2-6}$

- Task-shifting from clinical officers to nurses as a standalone intervention was not effective at improving ART initiation rates. ${ }^{7}$

- Comprehensive service delivery models that reduced client visits, improved familycentered care, offered multi-disease services, and home-based follow-up were effective at increasing ART initiation among children. ${ }^{8-11}$

\section{Retention/adherence as an outcome}

Total number of included studies: 36

Populations targeted: infants (11); children

(23); adolescents (28); and youth (11)

Study designs: non-experimental and no comparison group (21); non-experimental with a comparison group (9); and experimental (6)

\section{Effectiveness:}

- There was mixed evidence for the impact of education and counseling intervention models on retention in care and adherence among children and adolescents. ${ }^{12-14}$

- Case management models showed efficacy for care retention among adolescents in one quasi-experimental evaluation, ${ }^{15}$ and weaker evidence of efficacy in a study with a more rigorous randomized controlled trial (RCT). ${ }^{16}$

- There was good support for the effectiveness of phone-based outreach interventions to promote care retention among children and adolescents. ${ }^{17,18}$

- Electronic infant tracking system in Kenya ${ }^{19}$ and point-of-care PCR HIV testing for newborns were also effective at improving retention when evaluated in an $\mathrm{RCT}^{3}{ }^{3}$

- Decentralization or down-referral models improved retention outcomes among children and adolescents in multiple countries. ${ }^{20-22}$

- There was mixed evidence from cliniclevel interventions for improving retention and adherence outcomes. The majority of these interventions involved differentiated care models, with a focus on either family- or youth-friendly services, and most interventions indicated differentiated care was effective for either retention in care or adherence..$^{1,9,23-29}$ 


\section{Viral suppression as an outcome}

Total number of included studies: 12

Populations targeted: infants (2); children (9); adolescents (12); and youth (3)

Study designs: non-experimental and no comparison group (5); non-experimental with a comparison group (5); and experimental (2)

\section{Effectiveness:}

- Three studies documented significant reductions ${ }^{16,30,31}$ and one study found no difference in viral load ${ }^{32}$ through case management approaches using a mix of experimental and non-experimental designs among children, adolescents, and youth.

- The use of an illustrated book led to improved disclosure and viral suppression ${ }^{33}$ while a medication diary led to no change in viral suppression among a small sample of infants, children, and adolescents. ${ }^{34}$

- An economic empowerment intervention was evaluated with a cluster RCT that led to 10 times greater viral suppression among adolescents in the intervention group compared to the control group. ${ }^{35}$

- In an adolescent-friendly clinic, researchers observed improved viral load suppression among adolescents and youth after more than five years of follow-up. ${ }^{1}$

- In a transition to universal test and treat, children were less likely to be virally suppressed after six months of follow-up. ${ }^{36}$

\section{CONCLUSIONS}

This review identified a variety of effective strategies for improving engagement in HIV and ART adherence among children, adolescents, and youth living with HIV in sub-Saharan Africa. Approaches included phonebased reminder and tracing programs, group education and peer support, and expedited care delivery. Given this broad menu of options, the most appropriate strategy for any given context will likely need to be tailored to ensure it fits the specific objectives and target audience. Furthermore, to foster progress in generating evidence for future interventions, more rigorous study designs should be employed. 


\begin{tabular}{|c|c|c|}
\hline Study & Intervention & Effect size \\
\hline $\begin{array}{l}\text { Bermudez et } \\
\text { al., } 2018\end{array}$ & $\begin{array}{l}\text { Suubi+Adherence: A savings- } \\
\text { led economic empowerment } \\
\text { intervention on viral suppression } \\
\text { for adolescents ( } 10-16 \text { years) }\end{array}$ & $\begin{array}{l}\text { Proportion of virally suppressed } \\
\text { participants in the intervention } \\
\text { cohort increased tenfold }(\Delta \mathrm{T} 2- \\
\mathrm{TO}=+10.0, \mathrm{p}=0.001) \text { relative to } \\
\text { the control group }(\Delta \mathrm{T} 2-\mathrm{TO}=+1.1 \text {, } \\
\mathrm{p}=0.733) \text {. }\end{array}$ \\
\hline
\end{tabular}

Bigna et al., Mobile phone appt. reminders

2013, 2014 for infants, children and adolescents $(0-15$ years $)$
Appointment attendance (retention in care): OR 7.5 (95\% Cl 2.9-19.0; $\mathrm{p}<0.0001$ ) for call+SMS; OR 5.5 (2.3-13.1; $p=0.0002)$ for call; OR 2.9 for SMS $(1.3-6.3 ; p=0.012)$
Conclusion/recommendations

Interventions addressing economic insecurity have the potential to bolster health outcomes, such as HIV viral suppression, by improving ART adherence among vulnerable adolescents living in low resource environments.
Ferrand et al., 2017
ZENITH: Decentralized primary healthcare clinic-based HIV care plus structured support home visits for caregivers by trained community health workers targeting children and adolescents ( $6-15$ years)
Retention in care: aOR $0.92(95 \%$ Cl 0.49-1.74; $p=0.79$ )

Self-reported nonadherence: aOR $0.75(95 \% \mathrm{Cl} 0.24-2.35 ; \mathrm{p}=0.62)$

Viral suppression: aOR $0.46(95 \%$ Cl 0.23-0.89; $p=0.02$ )
The most effective method of appointment reminder was a text message followed by a phone call, but a text message alone was the most cost-effective method though the use of text messaging alone significantly improved attendance at the appointment compared with control.
The case management intervention improved viral suppression in children and adolescents, however, a substantial proportion in the intervention group still had poor outcomes leading the authors to conclude that It is unlikely that one intervention will suffice to achieve optimum outcomes. To scale up community-based interventions, careful attention needs to be paid to training and mentoring community workers, addressing contextual issues, and monitoring for quality assurance.

Finocchario- HIV Infant Tracking System Kessler et (HITSystem): a web-based al., 2018 intervention linking providers, laboratory technicians, and mothers and infants for quality improvement of early infant diagnosis (EID)

Graves et al., Family Clinic Day (FCD) 2018 differentiated care model including: patient scheduling, health education, patient flow prioritizing children, and their families for care over other patients (19 months to 19 years)

$\begin{array}{ll}\text { Jani et al., } & \text { Point-of-care (POC) early infant } \\ 2018 & \text { HIV testing }\end{array}$

Linnemayr et SMS reminder messages on al., 2017 antiretroviral and cotrimoxazole prophylaxis adherence targeting adolescents and youth (15-22 years)
ART initiation: $100 \%$ of $21 \mathrm{HIV}$ positive infants at intervention sites initiated ART ( $73 \%$ of 11 HIVpositive infants at control sites)

Retention in care: aOR $3.7(95 \% \mathrm{Cl}$ 2.5-5.5; $p<0.0001$ )

Retention in care (at least 1 appt in the last 3 months of study): aOR 1.11; (90\% Cl 0.63-1.97; $\mathrm{p}=0.75$ )

Adherence to last appointment with sufficient ARV: aOR 1.64 (90\% Cl 1.27-2.11; $p<0.001$ )

ART initiation: aRR 7.34, $(95 \% \mathrm{Cl}$ 4.7-11.5; $p<0.001$ )

Retention in care: aRR 1.40 $(p<0.027)$

Adherence $67 \%$ in control group, $64 \%$ in SMS only, $61 \%$ in message + response option; $\mathrm{p}=0.35$.
Implementation of the HITSystem was associated with more than a two-fold increase in both EID retention and initiation of ART among infants living with HIV compared to historical controls. The HITSystem's integration of customized alerts (Internet and SMS-based) with dedicated prospective tracking proved to be feasible and effective in both settings (urban, peri-urban) and in both types of hospitals (large maternity and district).

The FCD did not improve retention but was associated with improved adherence to last appointment scheduled. Patient health outcomes may benefit following an increase in knowledge as a result of health education, and peer support. Broad challenges facing ART clinics, such as under-staffing and poor filing systems, should be addressed in order to improve patient care.

POC infant HIV testing enabled clinics to more rapidly diagnose and provide treatment to HIV-infected infants. This reduced opportunities for pretreatment loss to follow-up and enabled a larger proportion of infants to receive test results and initiate antiretroviral therapy. The benefits of faster HIV diagnosis and antiretroviral treatment may also improve early retention in care.

SMS reminder messages did not have an effect on multiple measures adherence to ART. Simple reminder messages may not be enough to capture the attention of recipients, and considering other functions of SMS messages such as communicating adherence feedback, sending small incentives, or integration into a more involved mHealth approach may lead to more fruitful future interventions. 


\section{REFERENCES}

1. Zanoni, B.C. et al. 2017. "Higher retention and viral suppression with adolescent-focused HIV clinic in South Africa," PLoS One 12(12): e0190260. doi: 10.1371/journal. pone.0190260

2. Finocchario-Kessler, S. et al. 2014. "If you text them, they will come: using the HIV infant tracking system to improve early infant diagnosis quality and retention in Kenya," AIDS 28(Suppl 3): S313-S321. doi: 10.1097/ QAD.0000000000000332

3. Jani, I.V. et al. 2018. "Effect of point-of-care early infant diagnosis on antiretroviral therapy initiation and retention of patients," AIDS 32(11): 1453-1463. doi: 10.1097/ QAD.0000000000001846

4. Mwenda, R, et al. 2018. "Significant patient impact observed upon implementation of point-of-care early infant diagnosis technologies in an observational study in Malawi," Clinical Infectious Diseases 67(5): 701-707. doi: 10.1093/ cid/ciy169

5. Technau, K.G. et al. 2017. "Xpert HIV-1 point-of-care test for neonatal diagnosis of HIV in the birth testing programme of a maternity hospital: a field evaluation study," Lancet HIV 4(10): e442-e448. doi: 10.1016/S2352-3018(17)30097-8

6. Tejiokem, M.C. et al. 2015. "Feasibility of routinely offering early combined antiretroviral therapy to HIV-infected infants in a resource-limited country: the ANRS-PediaCAM Study in Cameroon," Pediatric Infectious Disease Journal 34(10): e248-253. doi: 10.1097/INF.0000000000000815

7. Marotta, C. et al. 2018. "Pathways of care for HIV infected children in Beira, Mozambique: pre-post intervention study to assess impact of task shifting," BMC Public Health 18(1): 703. doi: 10.1186/s12889-018-5646-8

8. Luyirika, E. et al. 2013. "Scaling up paediatric HIV care with an integrated, family-centred approach: an observational case study from Uganda," PLoS One. 8(8): e69548. doi: 10.1371/journal.pone.0069548

9. Gamell, A. et al. 2016. "Implementation and operational research: An integrated and comprehensive service delivery model to improve pediatric and maternal HIV care in rural Africa," Journal of Acquired Immune Deficiency Syndromes 73(5): e67-e75. doi: 10.1097/QAl.0000000000001178

10. Kose, J. et al. 2018. "Impact of a comprehensive adolescent-focused case finding intervention on uptake of HIV testing and linkage to care among adolescents in western Kenya," Journal of Acquired Immune Deficiency Syndromes 79(3): 367-374. doi: 10.1097/ QAI.0000000000001819

11. Ruria, E.C. et al. 2017. "Optimizing linkage to care and initiation and retention on treatment of adolescents with newly diagnosed HIV infection," AIDS 31(Suppl 3): S253s260. doi: 10.1097/QAD.0000000000001538

12. Bhana, A. et al. 2014. "The VUKA family program: piloting a family-based psychosocial intervention to promote health and mental health among HIV infected early adolescents in South Africa," AIDS Care 26(1): 1-11. doi: 10.1080/09540121.2013.806770

13. Strasser, S, and S. Gibbons. 2014. "The development of HIV-related mental health and psychosocial services for children and adolescents in Zambia: the case for learning by doing," Children and Youth Services Review 45: 150-157. doi: 10.1016/j.childyouth.2014.03.032
14. Grimwood, A. 2012. "Community adherence support improves programme retention in children on antiretroviral treatment: a multicentre cohort study in South Africa," Journal of the International AIDS Society 15(2): 17381. doi: 10.7448/IAS.15.2.17381

15. Fatti, G. et al. 2018. "The effectiveness and costeffectiveness of community-based support for adolescents receiving antiretroviral treatment: an operational research study in South Africa," Journal of the International AIDS Society 21(Suppl 1): e25041. doi: 10.1002/jia2.25041.

16. Ferrand, R.A. et al. 2017. "The effect of community-based support for caregivers on the risk of virological failure in children and adolescents with HIV in Harare, Zimbabwe (ZENITH): an open-label, randomised controlled trial," Lancet Child \& Adolescent Health 1(3): 175-183. doi: 10.1016/S2352-4642(17)30051-2

17. Bigna, J.J. et al. 2014. "Effect of mobile phone reminders on follow-up medical care of children exposed to or infected with HIV in Cameroon (MORE CARE): a multicentre, single-blind, factorial, randomised controlled trial," Lancet Infectious Diseases 14(7): 600-608. doi: 10.1016/S14733099(14)70741-8

18. Fernandez-Luis, S. et al. 2018. "Reengagement of HIVinfected children lost to follow-up after active mobile phone tracing in a rural area of Mozambique," Journal of Tropical Pediatrics 65(3): 240-248. doi: 10.1093/tropej/fmy041

19. Finocchario-Kessler, S. et al. 2018. "Evaluation of the HIV Infant Tracking System (HITSystem) to optimise quality and efficiency of early infant diagnosis: a cluster-randomised trial in Kenya," Lancet HIV 5(12): e696-e705. doi: 10.1016/S2352-3018(18)30245-5

20. Fayorsey, R.N. et al. 2013. "Decentralization of pediatric HIV care and treatment in five sub-Saharan African countries," Journal of Acquired Immune Deficiency Syndromes 62(5): e124-130. doi: 10.1097/QAl.0b013e3182869558

21. Auld, A.F. et al. 2016. "Decentralizing access to antiretroviral therapy for children living with HIV in Swaziland," Pediatric Infectious Disease Journal 35(8): 886-893.

22. Makadzange, A.T. et al. 2018. "Temporal improvements in long-term outcome in care among HIV-infected children enrolled in public antiretroviral treatment care: an analysis of outcomes from 2004 to 2012 in Zimbabwe," Pediatric Infectious Disease Journal 37(8): 794-800. doi: 10.1097/ INF.0000000000001903

23. Graves, J.C. et al. 2018. "Impact of a Family Clinic Day intervention on paediatric and adolescent appointment adherence and retention in antiretroviral therapy: a cluster randomized controlled trial in Uganda," PLoS One 13(3): e0192068. 10.1371/journal.pone.0192068

24. Kwarisiima, D. et al. 2017. "High rates of viral suppression in adults and children with high CD4+ counts using a streamlined ART delivery model in the SEARCH trial in rural Uganda and Kenya," Journal of the International AIDS Society 20(Suppl 4): 21673. doi: 10.7448/IAS.20.5.21673

25. Bacha, J.M. et al. 2018. "The Standardized Pediatric Expedited Encounters for ART Drugs Initiative (SPEEDI): description and evaluation of an innovative pediatric, adolescent, and young adult antiretroviral service delivery model in Tanzania," BMC Infectious Diseases 18(1): 448. doi: 10.1186/s12879-018-3331-2 
26. Willis, N. et al. 2018. "Zvandiri-Bringing a differentiated service delivery program to scale for children, adolescents, and young people in Zimbabwe," Journal of Acquired Immune Deficiency Syndromes 78(Suppl 2): s115-s123. doi: 10.1097/QAI.0000000000001737

27. Fiori, K. et al. 2016. "Closing the delivery gaps in pediatric HIV care in Togo, West Africa: using the care delivery value chain framework to direct quality improvement," AIDS Care 28(Suppl 2): 29-33. doi: 10.1080/09540121.2016.1176678

28. Teasdale, C.A. et al. 2016. "Impact of youth and adolescent friendly services on retention of 10-24-year-olds in HIV care and treatment programs in Nyanza, Kenya," Journal of Acquired Immune Deficiency Syndromes 71(2): e56-59. doi: 10.1097/QAl.0000000000000877

29. Izudi, J, et al. 2018. "Retention of HIV-positive adolescents in care: a quality improvement intervention in mid-western Uganda," BioMed Research International 2018: 1524016. doi: 10.1155/2018/1524016

30. Anigilaje, E.A. et al. 2014. "Effects of leisure activities and psychosocial support on medication adherence and clinic attendance among children on antiretroviral therapy," HIV AIDS (Auckl). 6: 127-137. doi: 10.2147/HIV.S64964

31. Fatti, G, N. Shaikh, B. Eley, and A. Grimwood. 2014. "Improved virological suppression in children on antiretroviral treatment receiving community-based adherence support: A multicentre cohort study from South Africa," AIDS Care 26(4): 448-453. doi: 10.1080/09540121.2013.855699

32. Amzel, A, et al. 2018. "Community-based interventions to reach 95-95-95 for children and adolescents: an exploratory programmatic review from Lesotho," Journal of Acquired Immune Deficiency Syndromes 78: S81-S87. doi: 10.1097/ QAI.0000000000001735
33. Beima-Sofie, K.M. et al. 2017, "Pediatric HIV disclosure intervention improves knowledge and clinical outcomes in HIV-infected children in Namibia," Journal of Acquired Immune Deficiency Syndromes 75(1): 18-26. doi: 10.1097/ QAI.0000000000001290

34. Kannigan, Y, K.B. Spicer, and F. Naby. 2018. "The use of illustrated medication diaries to improve outcomes for children initiated on highly active antiretroviral therapy," South African Journal of HIV Medicine 19(1). doi: 10.4102/ sajhivmed.v19i1.804

35. Bermudez, L.G. et al. 2018. "Does economic strengthening improve viral suppression among adolescents living with HIV? Results from a cluster randomized trial in Uganda," AIDS and Behavior 22(11): 3763-3772. doi: 10.1007/ s10461-018-2173-7

36. Kabogo, J. et al. 2018. "Evidence of reduced treatment adherence among HIV infected paediatric and adolescent populations in Nairobi at the onset of the UNAIDS Universal Test and Treat Program," BMC Research Notes 11(1): 134. doi: 10.1186/s13104-018-3205-0

Suggested citation: Project SOAR. 2021. "Overview: Literature review of linkage, adherence, and retention interventions for seropositive children and young people in Africa," Project SOAR Results Brief. Washington, DC: Population Council.
Project SOAR is a six-year+ (September 2014-January 2021) cooperative agreement funded by the U. S. President's Emergency Plan for AIDS Relief and the U. S. Agency for International Development (Agreement No. AIDOAA-A-14-00060). The contents of this brief are the sole responsibility of Project SOAR and Population Council and do not necessarily reflect the views of PEPFAR, USAID, or the United States Government.

Population Council leads the Project SOAR consortium in collaboration with Avenir Health, Elizabeth Glaser Pediatric AIDS Foundation, the Johns Hopkins University, Palladium, and The University of North Carolina at Chapel Hill.
Project SOAR/Population Council 4301 Connecticut Avenue, NW, Suite 280 Washington, DC 20008

Tel: +1 2022379400

e-mail: ProjectSOAR@popcouncil.org projsoar.org

(c) Population Council, January 2021 